\title{
Kinematic synthesis of over-constrained double-spherical six-bar mechanism
}

\author{
Omar W. Maaroof ${ }^{\mathrm{a}, *}$, Mehmet İsmet Can Dede ${ }^{\mathrm{b}, 1}$ \\ a Department of Mechatronics Engineering, Mosul University, 41002 Mosul, Iraq \\ b Department of Mechanical Engineering, İzmir Institute of Technology, 35430 İzmir, Turkey
}

\section{A R T I C L E I N F O}

\section{Article history:}

Received 4 June 2012

Received in revised form 4 July 2013

Accepted 28 October 2013

Available online 28 November 2013

\section{Keywords:}

Kinematic synthesis

Over-constrained mechanism

Double-spherical six-bar mechanism

Interpolation approximation

\begin{abstract}
A B S T R A C T
The main problem in the synthesis of any mechanism is the fact that the objective function of the mechanism, which will be synthesized, should be found and simplified by using appropriate algebraic method. Finding objective function and calculation process can become complicated especially when the number of design parameters is increased for the over-constrained mechanisms. A new technique for solving the kinematic synthesis of over-constrained double-spherical six-bar mechanism is developed and applied in this work. Interpolation approximation is used during synthesis procedure. A numerical example for the kinematic synthesis procedure is given to validate the theory in application.
\end{abstract}

(c) 2013 Elsevier Ltd. All rights reserved.

\section{Introduction}

One of the main problems in kinematic synthesis is finding the function generation, which describes a relation between input and output of the mechanism with a desired function complying with the constraint conditions. Several mathematical methods including algebraic method, complex numbers method, vector method, quaternion and bi-quaternion methods, matrix and screw methods, and computer-aided-design (CAD) based methods have been developed and applied in order to solve for the kinematic synthesis problem. The kinematic synthesis problem becomes harder to solve for when the number of design parameters is increased especially for the over-constrained mechanisms.

Spherical mechanisms hold a transition position between the planar and spatial linkages, hence there is still an ongoing interest in solving for the kinematic synthesis of these mechanisms. Many studies have been conducted based on the limit positions of the input and output links. The design of spherical four-bar linkages for four specified orientations were was proposed by Ruth and McCarthy [1], where they described a CAD software solution procedure for spherical four-bar linkages that is based on Burmester's planar theory [2]. Cervantes-Sanchez and Medellin-Castillo [3,4] proposed a classification scheme and an improved motion analysis for spherical four-bar linkages. Shih-Hsi Tong and C.H. Chiang [5] discussed the syntheses of planar and spherical four-bar path generators, wherein they based the syntheses on the geometrical relations between the pole of the coupler and the joints of the mechanism.

Function generation synthesis of four-bar spherical linkage has been widely studied. Function generation for the entire motion cycle for spherical four-bar linkages has been discussed in [6]. Denavit and Hartenberg [7] presented the synthesis procedure for three precision points in the function generation of spherical four-bar mechanism. Zimmerman [8] proposed a different algorithm for the same mechanism for four precision points. Polynomial approximation is used for three, four and five precision points in the work of Murray and McCarthy [9], Alizade [10], and Alizade and Kilit [11], respectively for the spherical four-bar mechanism.

\footnotetext{
* Corresponding author. Tel.: + 9647702044149.

E-mail addresses: omarmaaroof@uomosul.edu.iq (O.W. Maaroof), candede@iyte.edu.tr (M.İ.C. Dede).

${ }^{1}$ Tel.: +902327506778.
} 
Furthermore, Alizade and Gezgin [12] proposed a new function generation synthesis method for spherical four-bar mechanism with six independent parameters. They applied interpolation approximation, least-square approximation and Chebyshev approximation and evaluated the error magnitudes of the approximations. Furthermore, several researchers made significant contributions in this field and many mathematical techniques have been developed for the synthesis and analysis of spherical four-bar mechanisms [14-17].

Double-spherical six-bar mechanism is not a new mechanism and researchers have identified simple constraints to have the mechanism move. Some have named the mechanism as double-Hooke's-joint linkage [19] or 6R double-spherical over-constrained mechanism [20] and worked on the analysis of the mechanism. In the study of N. Makhsudyan et al. [13], the comparative analysis and synthesis of six-bar mechanisms are discussed for two serially connected spherical four-bar linkages and two serially connected planar four-bar linkages. However, they placed the input and output axes of the spherical linkages parallel and the construction parameters of the first spherical and planar mechanism are selected by the authors and not determined by the kinematic synthesis procedure.

The objective of this study is the function generation synthesis of double spherical six-bar mechanism. A passive revolute joint is inserted into the mechanism between the intersections of the joint axes of the two spherical four-bars. The passive joint's rotation axis is aligned with the line drawn from one intersection point to the other one. The objective functions for the first and the second spherical four-bar are determined. The objective function of the double-spherical six-bar mechanisms is defined. However, the objective function of the first spherical four-bar linkage is selected independent of the whole mechanism's function between the input and the output. Four precision points are selected by using equal spacing for the first spherical four-bar linkage and the double-spherical six-bar mechanism. Interpolation approximation method is used for designing the six construction parameters for the mechanism and the results are tabulated.

The next section of this paper provides the theory for the synthesis of six-bar mechanisms. Structural synthesis of double-spherical six-bar mechanism is explained through the analogy between various planar and spatial six-bar mechanisms. Later, the kinematic synthesis procedure for function generation of the double-spherical six-bar mechanism is explained. Objective function determination and interpolation approximation methods are then complemented by a numerical example to validate the theory presented in this paper. Finally, discussions on the developed methodology and the results are given in the last section.

\section{Structural synthesis of six-bar mechanisms}

This section consists of the analogy between the structural syntheses of six-bar mechanisms. The first step in the design of new robot manipulators is structural synthesis, which is the fundamental concept in mechanism design. In this section, structural synthesis of three 6R spatial closed-loop serial robot manipulators is considered.

The mobility of a robot mechanism describes the number of actuators needed to define the location of end-effectors. It is important that the mobility or the degrees of freedom (DoF) of robot manipulators $(\mathrm{M}>1)$ indicates the number of independent input parameters to determine all the possible configurations of robot manipulators.

If links are connected in a loop by four single-DoF joints and the joints are configured so that the links move in parallel planes, the assembly is called a planar four-bar linkage [7]. If the linkage has four revolute joints with axes angled to intersect in a single point, then the links move on concentric spheres, and thus, the assembly is called a spherical four-bar linkage.

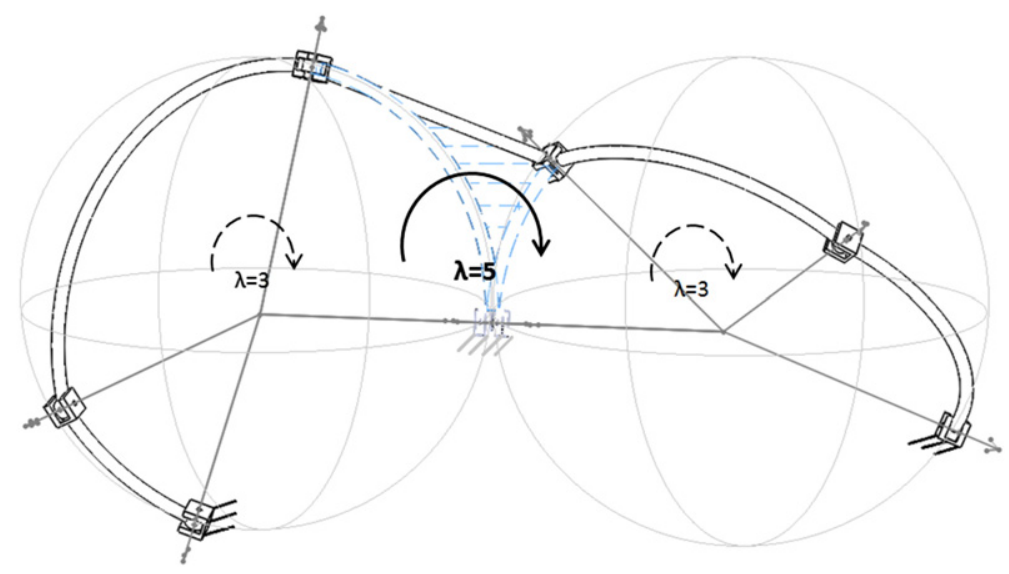

Fig. 1. $(R R R)(R R R)$ double-spherical six-bar mechanism. 
Alizade et al. [18] generalized Freudenstein's equation [22] on determining the DoF or mobility equation for robotic systems with independent loops and variable general constraint as presented in Eq. (1).

$$
M=-L \lambda+\sum_{i=1}^{j} f_{i}+q-j_{p}
$$

In Eq. (1), $\lambda$ is the general constraint parameters of simple structural groups, $L=c-B$ is the number of independent loops, $c=c_{b}+c_{l}+c_{h}\left(c_{b}\right.$ is the number of branches between platforms, $c_{h}$ is the number of hinges and $c_{l}$ is the number of legs), $B$ is the number of moving platforms, $j_{p}$ is number of passive joints, $q$ is the number of excessive links or overclosing constraints, $f_{i}$ is DoF of relative motion permitted at the $i$ th joint and $j$ is the number of joints.

The general constraint parameters of the simple structural groups can be calculated if the mobility is selected for a mechanism by pre-determining the structural configuration of the mechanism as shown in Eq. (2).

$$
\lambda=\frac{\sum_{i=1}^{j} f_{i}+q-j_{p}-M}{L}
$$

For the double spherical six-bar linkage presented in Fig. 1, Eq. (2) is applied as the mobility of the mechanism is known to be $M=1$, and the general constraint parameters of the simple structural group is calculated to be $\lambda=5$. In order to accommodate the kinematic synthesis, the double-spherical six-bar mechanism is required to be redefined as a serially connected two spherical four-bar mechanisms. Therefore, a passive joint with excessive connections is inserted between the two spherical linkages. The location of the passive joint is between the intersection of the joint axes and the direction of the passive joint axis is aligned with the line drawn from one intersection point to the other one. As a result of this, there forms an excessive platform $B=1$ with $c=$ 3 , hence $L=2$, total DoF of all joints is increased to 7, mobility is required to be one, $M=1$, also $q=1$ and $j_{p}=1$. Substituting this information in Eq. (2), $\lambda$ is calculated to be 3 for each independent loop. Therefore, it is validated that for the same mechanism without the insertion of the passive joint and excessive connections ( $L=1$, total DoF of joints is $6, M=1$, also $q=0$ and $j_{p}=0$ ), $\lambda$ is calculated to be 5 using Eq. (2) for the double-spherical six-bar mechanism. The structural synthesis procedure of the planar-spherical 6R linkage presented in Fig. 2 and the spatial planar-planar 6R linkage shown in Fig. 3 is analogous to the structural synthesis procedure of the double-spherical six-bar linkage. The general constraint parameter of the simple structural group for both mechanisms is calculated to be $\lambda=5$. When both mechanisms are reconfigured to have two closed loops, the general constraint parameter of the simple structural group for each loop is calculated to be $\lambda=3$. It should be noted that for the planar-planar 6R linkage, a passive prismatic joint is inserted instead of a passive revolute joint.

\section{Double-spherical six-bar mechanism}

In this section, mechanism is described and mechanism parameters are reconfigured as two spherical four-bar mechanisms that are connected with a passive joint. Input and output equations for both the first spherical linkage and the second spherical linkage are provided.

\subsection{Description of the linkage}

Double-spherical linkage is a mechanism with general constraint one, which belongs to subspace $\lambda=5$. It has six revolute joints whose axes are intersecting three by three in two different points. The screw axes (joint unit vectors) that denote the two

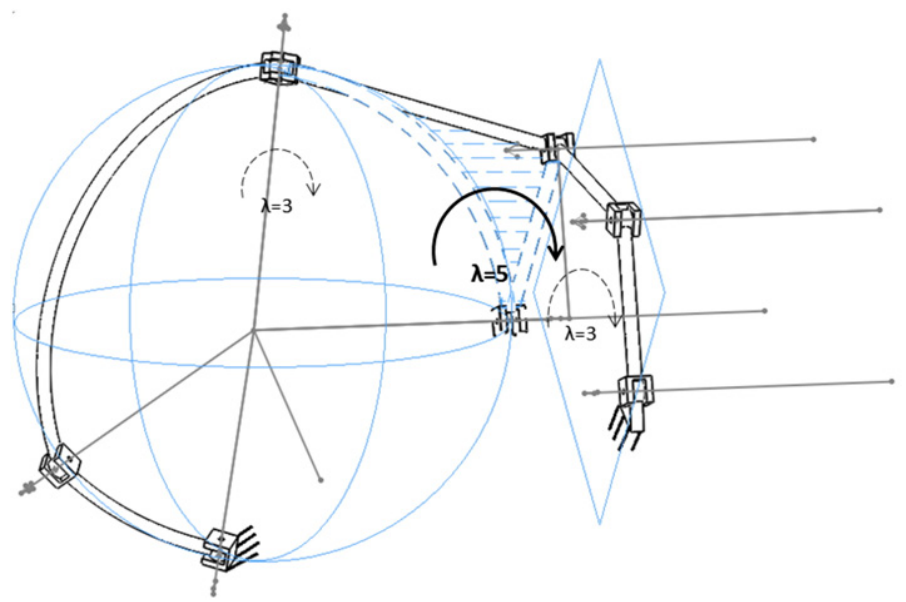

Fig. 2. $(R R R)(\overline{R R R})$ spherical-planar six-bar mechanism. 


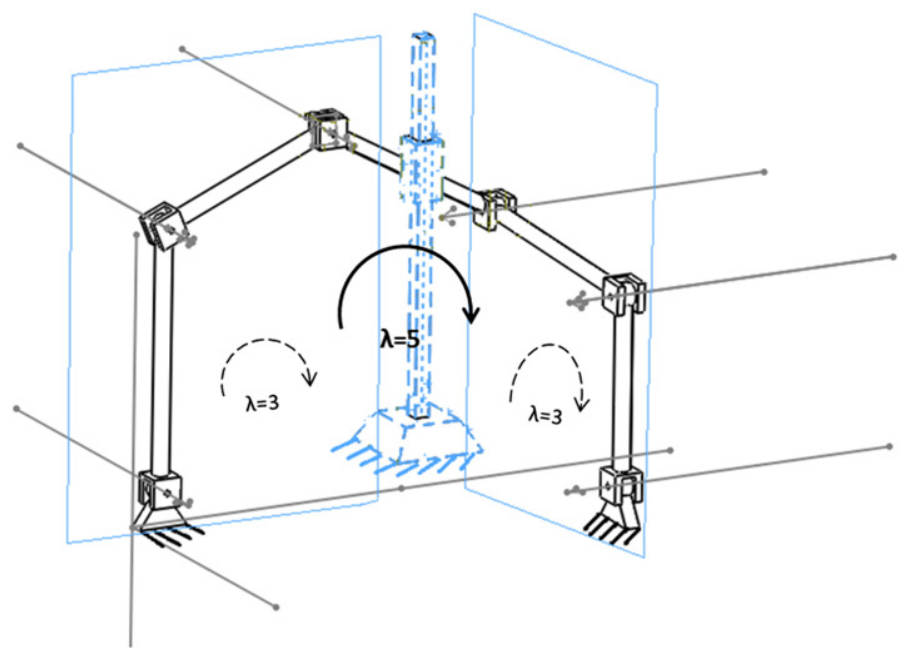

Fig. 3. $\overline{R R R} \underline{R R R}$ planar-planar spatial six-bar mechanism.

groups of mechanisms are $\mathbf{S}_{14}-\mathbf{S}_{12}-\mathbf{S}_{10}$ and $\mathbf{S}_{6}-\mathbf{S}_{4}-\mathbf{S}_{2}$. For the input-output function synthesis of the double spherical linkage, the mechanism is described as two spherical four-bar linkages as shown in Fig. 4. The passive revolute joint that has the $\mathbf{S}_{8}$ rotation axis is added to the mechanism. This passive joint is included in both groups to form two separate spherical four-bar mechanisms as $\mathbf{S}_{14}-\mathbf{S}_{12}-\mathbf{S}_{10}-\mathbf{S}_{8}$ and $\mathbf{S}_{8}-\mathbf{S}_{6}-\mathbf{S}_{4}-\mathbf{S}_{2}$.

The transformation unit vector equation described in [21] can be used as a valuable tool to relate the unit vectors assigned in Fig. 4. Therefore, for each $\mathbf{S}_{i}\left(l_{i}, m_{i}, n_{i}\right)$ that rotates around $\mathbf{S}_{j}\left(l_{j}, m_{j}, n_{j}\right)$ by angle $\alpha_{i k}$, we can calculate $\mathbf{S}_{k}\left(l_{k}, m_{k}, n_{k}\right)$ as follows;

$$
\boldsymbol{S}_{k}=\boldsymbol{S}_{i} \cos \alpha_{i k}+\boldsymbol{S}_{j i} \sin \alpha_{i k}
$$

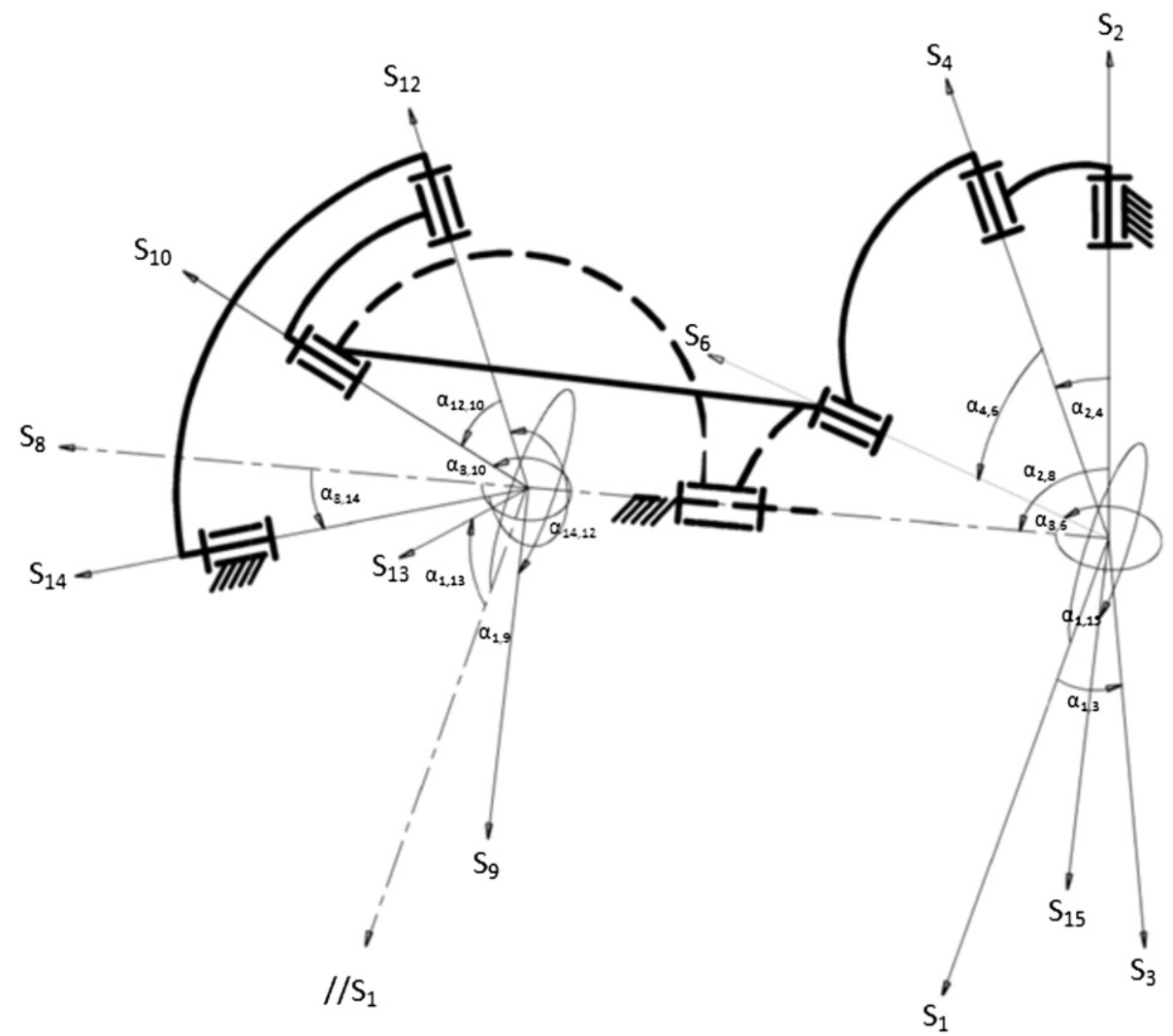

Fig. 4. The double-spherical mechanism with screw axes. 
where,

$$
\boldsymbol{S}_{j i}=\boldsymbol{S}_{j} \times \boldsymbol{S}_{i} .
$$

The screws of revolute pairs of double-spherical six-bar mechanism were shown as joint unit vectors, where $\mathbf{S}_{1}=(1,0,0)$ and $\mathbf{S}_{2}=(0,0,1)$. Finding the other screws is important to find the objective functions of the two four-bar linkages. So we find the screws as follows:

\begin{tabular}{ll}
\hline For the first four-bar linkage of the mechanism & For the second four-bar linkage of the mechanism \\
\hline $\mathbf{S}_{1}, \mathbf{S}_{2}$ and $\alpha_{1,3} \rightarrow \mathbf{S}_{3}$, & $\mathbf{S}_{8}, \mathbf{S}_{1}$ and $\alpha_{8,14} \rightarrow \mathbf{S}_{14}$ \\
$\mathbf{S}_{2}, \mathbf{S}_{3}$ and $\alpha_{2,4} \rightarrow \mathbf{S}_{4}$, & $\mathbf{S}_{1}, \mathbf{S}_{14}$ and $\alpha_{1,13} \rightarrow \mathbf{S}_{13}$ \\
$\mathbf{S}_{2}, \mathbf{S}_{1}$ and $\alpha_{2,8} \rightarrow \mathbf{S}_{8}$, & $\mathbf{S}_{14}, \mathbf{S}_{13}$ and $\alpha_{14,12} \rightarrow \mathbf{S}_{12}$ \\
$\mathbf{S}_{1}, \mathbf{S}_{8}$ and $\alpha_{1,15} \rightarrow \mathbf{S}_{15}$, & $\mathbf{S}_{1}, \mathbf{S}_{8}$ and $\alpha_{1,9} \rightarrow \mathbf{S}_{9}$ \\
$\mathbf{S}_{8}, \mathbf{S}_{15}$ and $\alpha_{8,6} \rightarrow \mathbf{S}_{6}$, & $\mathbf{S}_{8}, \mathbf{S}_{9}$ and $\alpha_{8,10} \rightarrow \mathbf{S}_{10}$ \\
\hline
\end{tabular}

\subsection{Input-output equation of the first spherical linkage}

The objective function (input-output equation) of the first four-bar linkage will be calculated as ( $\mathrm{C} \alpha=\cos \alpha \& \mathrm{~S} \alpha=\sin \alpha$ ):

$$
\boldsymbol{S}_{6} \cdot \boldsymbol{S}_{4}=\mathrm{C} \alpha_{4,6} .
$$

Substituting values of $\mathbf{S}_{4}$ and $\mathbf{S}_{6}$ and making some arrangements will result in:

$$
-\mathrm{C} \alpha_{4,6}+C \alpha_{2,4} \mathrm{C} \alpha_{2,8} \mathrm{C} \alpha_{8,6}+C \alpha_{1,3} \mathrm{C} \alpha_{8,6} \mathrm{~S} \alpha_{2,4} \mathrm{~S} \alpha_{2,8}+\mathrm{C} \alpha_{1,3} \mathrm{C} \alpha_{1,15} \mathrm{C} \alpha_{2,8} \mathrm{~S} \alpha_{2,4} \mathrm{~S} \alpha_{8,6}-\mathrm{C} \alpha_{1,15} \mathrm{C} \alpha_{2,4} \mathrm{~S} \alpha_{2,8} \mathrm{~S} \alpha_{8,6}+\mathrm{S} \alpha_{1,3} \mathrm{~S} \alpha_{1,15} \mathrm{~S} \alpha_{2,4} \mathrm{~S} \alpha_{8,6}=0
$$

where the input $\phi$ is $\alpha_{1,3}$ and output $\widetilde{\psi}$ is $\alpha_{1,15}$.

The function can be written in the polynomial form if divided by $-S \alpha_{2,4} S \alpha_{8,6}$ as;

$$
P_{0} f_{0}(\phi)+P_{1} f_{1}(\phi)+P_{2} f_{2}(\phi)+P_{3} f_{3}(\phi)-F(\phi)=0
$$

where

$$
\begin{aligned}
& P_{0}=\left(C \alpha_{4,6}-C \alpha_{2,4} C \alpha_{2,8} C \alpha_{8,6}\right) /\left(S \alpha_{2,4} S \alpha_{8,6}\right), P_{1}=-C \alpha_{8,6} S \alpha_{2,8} / S \alpha_{8,6}, P_{2}=-C \alpha_{2,8}, \\
& P_{3}=C \alpha_{2,4} S \alpha_{2,8} / S \alpha_{2,4} \\
& f_{0}(\phi)=1, \quad f_{1}(\phi)=C \phi, \quad f_{2}(\phi)=C \phi C \widetilde{\psi}, \quad f_{3}(\phi)=C \widetilde{\psi}, \quad F(\phi)=S \phi S \widetilde{\psi} .
\end{aligned}
$$

\subsection{Input-output equation of the second spherical linkage}

The objective function (input-output equation) for the second four-bar linkage will be:

$$
\boldsymbol{S}_{12} \cdot \boldsymbol{S}_{10}=C \alpha_{10,12}
$$

where the input $\widetilde{\psi}$ is $\alpha_{1,9}$ and output $\psi$ is $\alpha_{1,13}$.

The function can be written in the polynomial form if divided by $-S \alpha_{8,10} S \alpha_{14,12}$ as:

$$
R_{0} g_{0}(\widetilde{\psi})+R_{1} g_{1}(\widetilde{\psi})+R_{2} g_{2}(\widetilde{\psi})+R_{3} g_{3}(\widetilde{\psi})-G(\widetilde{\psi})=0
$$

where

$$
\begin{aligned}
& R_{0}=\left(C \alpha_{10,12}-C \alpha_{8,10} C \alpha_{8,14} C \alpha_{14,12}\right) /\left(S \alpha_{8,10} S \alpha_{14,12}\right), R_{1}=-C \alpha_{14,12} S \alpha_{8,14} / S \alpha_{14,12}, \\
& R_{2}=-C \alpha_{8,14}, R_{3}=C \alpha_{8,10} S \alpha_{8,14} / S \alpha_{8,10} \\
& g_{0}(\widetilde{\psi})=1, \quad g_{1}(\widetilde{\psi})=C \widetilde{\psi}, \quad g_{2}(\widetilde{\psi})=C \widetilde{\psi} C \psi, \quad g_{3}(\widetilde{\psi})=C \psi, \quad G(\widetilde{\psi})=S \widetilde{\psi} S \psi
\end{aligned}
$$

\section{Kinematic synthesis}

Synthesis of double-spherical six-bar mechanism is difficult since the number of construction parameters is high and objective function is hard to obtain. However, the mechanism can be separated into two sections and solved recurrently. The next sub-sections describe the Chebyshev spacing and precision points used in the synthesis procedure and derivation and computation of the design equations for both spherical four-bar linkages. 


\subsection{Chebyshev spacing and precision points}

It is assumed that there are two mechanisms and a virtual revolute pair between these two parts is included in the loop, where the output of the first one is the input for the second spherical four-bar linkage. Making use of having two successive mechanisms that use prior mechanism's output as the input for the second mechanism, function decomposition can be accommodated. A given objective function can be decomposed into function of a function and therefore, the objective function can be shared between two spherical four-bar linkages. The procedure for the function generation synthesis by decomposition method starts with selecting an overall double-spherical six-bar linkage function, function $y=f(x)$. Following this, an arbitrary function $\hat{y}=h(x)$ is selected as the first function. The range of $x$ is given as $x_{0} \leq x \leq x_{m}$ and respectively $\hat{y}_{0} \leq \hat{y} \leq \hat{y}_{m}$ where $\hat{y}_{0}=h\left(x_{0}\right)$ and $\hat{y}_{\mathrm{m}}=h\left(x_{m}\right)$.

Initially, the arbitrary function $\hat{y}=h(x)$ is scaled for the input $(\phi)$ and output $(\widetilde{\psi})$ of the first mechanism. When the range of input is $\phi_{0} \leq \phi \leq \phi_{m}$ and the range of output is selected as $\widetilde{\psi}_{0} \leq \widetilde{\psi} \leq \widetilde{\psi}_{m}$, the scaling equations will be;

$$
\phi=\hat{a}_{1} x+\hat{a}_{2}, \widetilde{\psi}=b^{\prime}{ }_{1}^{\prime} y+b^{\prime}{ }_{2}
$$

and the selected objective function for the output angle for the first mechanism will be;

$$
\widetilde{\psi}=b^{\prime}{ }_{1} h\left(\frac{\phi-\hat{a}_{2}}{\hat{a}_{1}}\right)+b_{2} .
$$

After defining the input-output relations, a generated function is found by making synthesis of the first four-bar linkage as;

$$
\widetilde{\psi}=h(\phi, \bar{c})
$$

where $\bar{c}$ is the vector containing the designed construction parameters of the first four-bar linkage.

After designing the construction parameters for the first four-bar linkages, the objective function for the second spherical four-bar linkage should be calculated. The calculation should be conducted to find a function $y=g(\hat{y})$, where $y=g(h(x))=$ $f(x)$. The range of input for the second spherical linkage is the same as the range of output of the first spherical linkage. The range of output should be selected as the range of output for the whole mechanism, $\psi_{0} \leq \psi \leq \psi_{m}$. Thus, the scale equations are;

$$
\widetilde{\psi}=b^{\prime}{ }_{1}^{\prime} y+b^{\prime}{ }_{2}, \psi=b_{1} y+b_{2}
$$

and the desired objective function for the output angle of the whole mechanism will be;

$$
\psi=b_{1} g\left(\frac{\widetilde{\psi}-b_{2}}{b_{1}^{\prime}}\right)+b_{2}
$$

After defining the input-output relations, a generated function is found by making synthesis of the second four bar linkages as;

$$
\psi=g(\widetilde{\psi}, \bar{d}) .
$$

Substituting Eq. (12) in Eq. (15), the generated function for the whole double-spherical six-bar mechanism can be calculated as;

$$
\psi=g(h(\phi, \bar{c}), \bar{d})
$$

where $\bar{c}$ and $\bar{d}$ are the designed construction parameters of the first and second four-bar linkages respectively.

Interpolation approximation will be used for finding the construction parameters of the mechanism. For the exact solution, 4 positions of the mechanism are required and four unknown parameters for each spherical linkage must be determined. Thus, $n=4$ (where $n$ is the number of equations or precision points) and precision points are distributed for exact synthesis equally in 


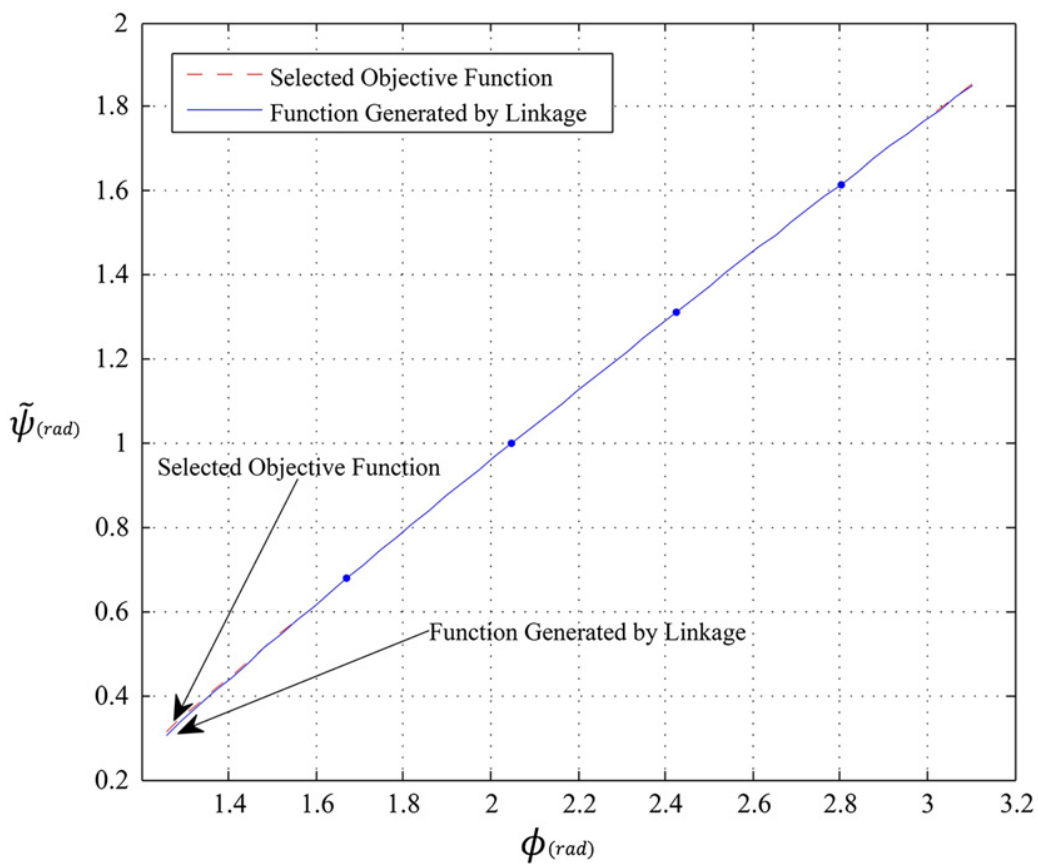

Fig. 5. Selected objective function, $\hat{y}=x^{0.8}$, and function generated by first linkage of example $\# 1$.

the range of $x$ from $x_{0}=1$ to $x_{m}=2$ as shown in Eq. (17).

$$
x_{n}=x_{n-1}+\delta \quad n=1,2,3,4
$$

When Eq. (18) is used for equal spacing, $\delta=0.2$.

$$
\delta=\left(x_{m}-x_{0}\right) /(n+1) \text { for } n=4
$$

$y$ values of the given and selected functions can be calculated by

$$
y_{1}=f\left(x_{i}\right), \quad i=0, n+1 .
$$

The scaled equations of input and output variables are given in Eq. (10), the constant parameters will be calculated using Eq. (20) for the first and second linkages.

$$
\begin{array}{ll}
a_{1}=\left(\phi_{0}-\phi_{m}\right) /\left(x_{0}-x_{m}\right), & a_{2}=\left(x_{m} \phi_{0}-x_{0} \phi_{m}\right) /\left(x_{m}-x_{0}\right) \\
b_{1}=\left(\psi_{0}-\psi_{m}\right) /\left(y_{0}-y_{m}\right), & b_{2}=\left(y_{m} \psi_{0}-y_{0} \psi_{m}\right) /\left(y_{m}-y_{0}\right)
\end{array}
$$

\subsection{Derivation of the first spherical linkage's design equation and computation of the design parameters}

General form of equations for the objective function shown in Eq. (21)

$$
\sum_{k=0}^{n-1} P_{k} f_{k}\left(\phi_{i}\right)-F\left(\phi_{i}\right)=0, \quad i=1, n
$$

Table 1

Precision points and designed construction parameters for the first spherical four-bar linkage of example \#1.

\begin{tabular}{rlllrr}
\hline$i$ & $x_{i}$ & $y_{i}$ & $\phi_{i}$ rad & $\bar{\psi}_{i}($ rad $)$ & $P_{i}$ \\
\hline 1 & 1.22 & 1.1724 & 1.6713 & 0.6796 & 0.2297 \\
2 & 1.42 & 1.3238 & 2.0483 & 1.0005 & -0.1702 \\
3 & 1.62 & 1.4710 & 2.4253 & 1.3125 & -0.9123 \\
4 & 1.82 & 1.6146 & 2.8023 & 1.6168 & $\alpha_{2,8}=0.4218$ \\
\hline
\end{tabular}




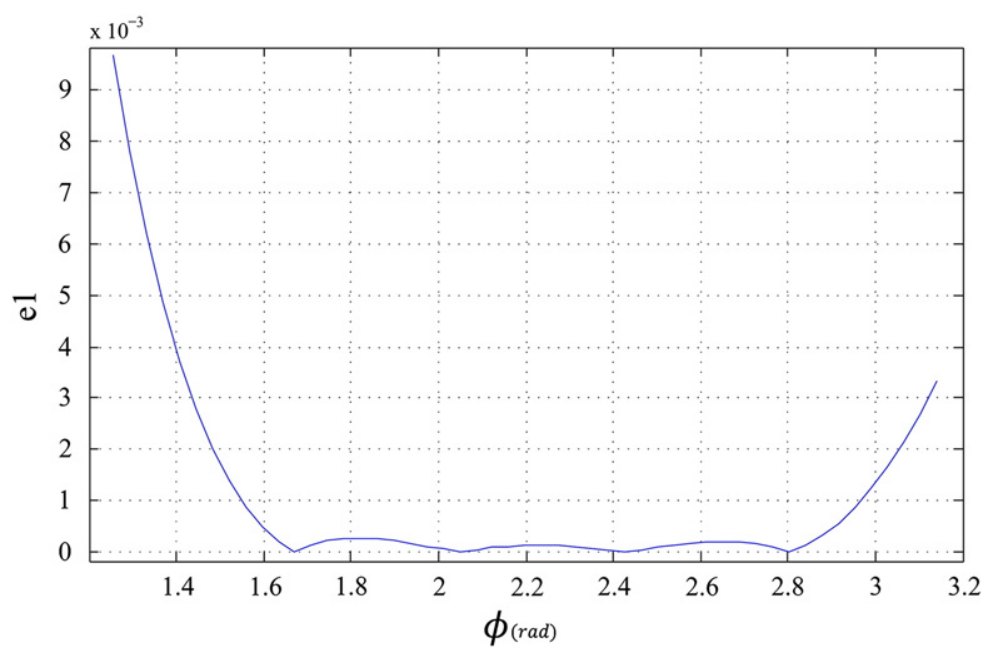

Fig. 6. Calculated error for the first spherical linkage of example \#1.

The number of precision points is four, thus, four linear equations with four unknowns are required for the objective function. The equations can be written in the matrix form,

$$
\left[\begin{array}{llll}
f_{0}\left(\phi_{1}\right) & f_{1}\left(\phi_{1}\right) & f_{2}\left(\phi_{1}\right) & f_{3}\left(\phi_{1}\right) \\
f_{0}\left(\phi_{2}\right) & f_{1}\left(\phi_{2}\right) & f_{2}\left(\phi_{2}\right) & f_{3}\left(\phi_{2}\right) \\
f_{0}\left(\phi_{3}\right) & f_{1}\left(\phi_{3}\right) & f_{2}\left(\phi_{3}\right) & f_{3}\left(\phi_{3}\right) \\
f_{0}\left(\phi_{4}\right) & f_{1}\left(\phi_{4}\right) & f_{2}\left(\phi_{4}\right) & f_{3}\left(\phi_{4}\right)
\end{array}\right] \cdot\left[\begin{array}{l}
P_{0} \\
P_{1} \\
P_{2} \\
P_{3}
\end{array}\right]=\left[\begin{array}{l}
F\left(\phi_{1}\right) \\
F\left(\phi_{2}\right) \\
F\left(\phi_{3}\right) \\
F\left(\phi_{4}\right)
\end{array}\right]
$$

Values of $P_{i}$ can be found by using Eq. (23) or by using Cramer's rule, and unknown construction parameters of the first spherical four-bar linkage can be determined as shown in Eq. (24).

$$
\begin{aligned}
& \bar{P}=\left[f_{i, j}\right]^{-1} \cdot \bar{F} \\
& \alpha_{2,8}=\cos ^{-1}\left(-P_{2}\right), \quad \alpha_{2,4}=\cot ^{-1}\left(\csc \left(\alpha_{2,8}\right) \cdot P_{3}\right), \quad \alpha_{8,6}=-\cot ^{-1}\left(\csc \left(\alpha_{2,8}\right) \cdot P_{1}\right) \\
& \alpha_{4,6}=\cos ^{-1}\left(\sin \left(\alpha_{2,4}\right) \cdot \sin \left(\alpha_{8,6}\right) \cdot\left(\cos \left(\alpha_{2,8}\right) \cdot \cot \left(\alpha_{2,4}\right) \cdot \cot \left(\alpha_{8,6}\right)+P_{0}\right)\right)
\end{aligned}
$$

Generated function can be calculated by using construction parameters determined in Eq. (24) and the input $\phi$ as in Eq. (12), where $\bar{c}=\left\{\alpha_{2,8}, \alpha_{2,4}, \alpha_{8,6}, \alpha_{4,6}\right\}$.

Here $\alpha$ angles are the angular link-length of spherical four-bar linkage as previously shown in Fig. 4. Eq. (12) will be used to find the input of the second spherical four-bar linkage.

\subsection{Derivation of the second spherical linkage's design equation and computation of the design parameters}

Similarly, for the second spherical four-bar linkage, the general form of equations derived from the objective function will be

$$
\sum_{k=0}^{n-1} R_{k} g_{k}\left(\widetilde{\psi}_{i}\right)-G\left(\widetilde{\psi}_{i}\right)=0, \quad i=1, n
$$

\begin{tabular}{|c|c|c|c|c|c|c|}
\hline$i$ & $\hat{y}_{i}$ & $y_{i}$ & $\bar{\psi}_{i}(\mathrm{rad})$ & $\psi_{i}(\mathrm{rad})$ & $R_{i}$ & Second four-bar parameters ( $\mathrm{rad}$ ) \\
\hline 1 & 1.1186 & 1.1998 & 0.5655 & 1.7377 & -0.4954 & $\alpha_{8,14}=0.8245$ \\
\hline 2 & 1.2668 & 1.4686 & 0.8797 & 1.9623 & 0.8375 & $\alpha_{14,12}=-0.7198$ \\
\hline 3 & 1.4150 & 1.7578 & 1.1938 & 2.2040 & -0.6789 & $\alpha_{8,10}=0.5035$ \\
\hline 4 & 1.5632 & 2.0667 & 1.5079 & 2.4620 & -1.3329 & $\alpha_{12,10}=0.9214$ \\
\hline
\end{tabular}

Table 2

Precision points and designed construction parameters for the second spherical four-bar linkage of example \#1. 


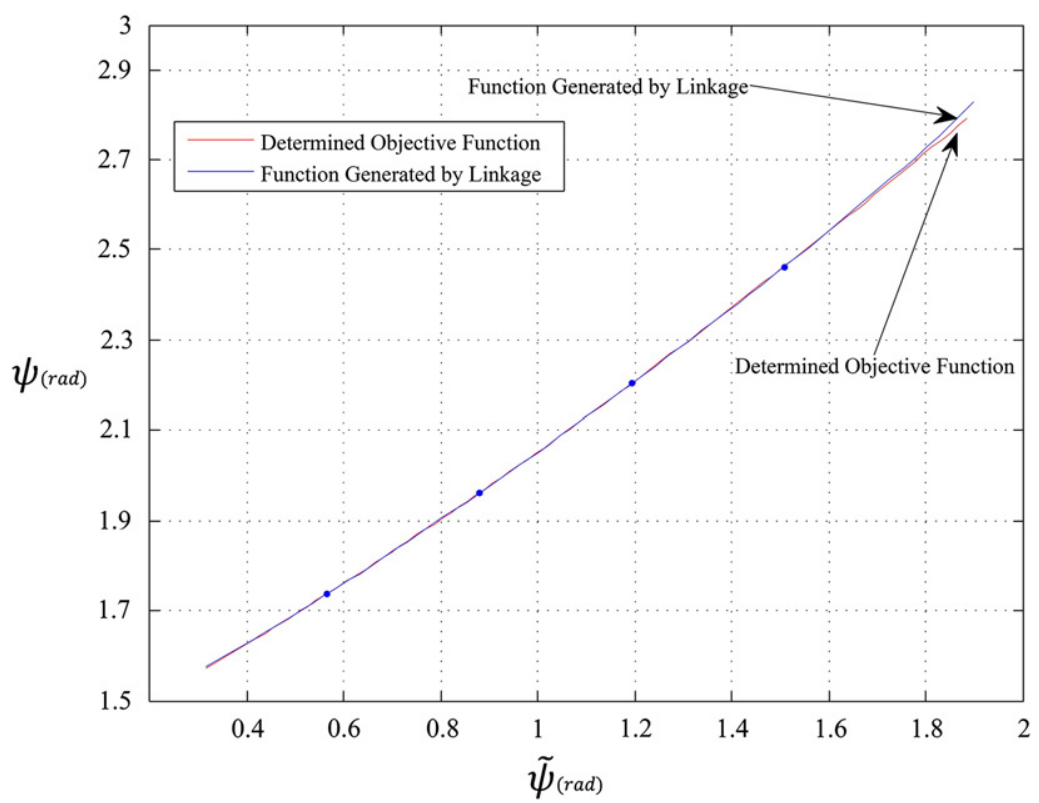

Fig. 7. Selected objective function, $y=y^{(1.625)}$, and function generated by second linkage of example \#1.

The number of precision points is again four, thus four linear equations with four unknowns are also acquired for the objective function and can be written in the matrix form,

$$
\left[\begin{array}{llll}
g_{0}\left(\widetilde{\psi}_{1}\right) & g_{1}\left(\widetilde{\psi}_{1}\right) & g_{2}\left(\widetilde{\psi}_{1}\right) & g_{3}\left(\widetilde{\psi}_{1}\right) \\
g_{0}\left(\widetilde{\psi}_{2}\right) & g_{1}\left(\widetilde{\psi}_{2}\right) & g_{2}\left(\widetilde{\psi}_{2}\right) & g_{3}\left(\widetilde{\psi}_{2}\right) \\
g_{0}\left(\widetilde{\psi}_{3}\right) & g_{1}\left(\widetilde{\psi}_{3}\right) & g_{2}\left(\widetilde{\psi}_{3}\right) & g_{3}\left(\widetilde{\psi}_{3}\right) \\
g_{0}\left(\widetilde{\psi}_{4}\right) & g_{1}\left(\widetilde{\psi}_{4}\right) & g_{2}\left(\widetilde{\psi}_{4}\right) & g_{3}\left(\widetilde{\psi}_{4}\right)
\end{array}\right] \cdot\left[\begin{array}{l}
R_{0} \\
R_{1} \\
R_{2} \\
R_{3}
\end{array}\right]=\left[\begin{array}{l}
G\left(\widetilde{\psi}_{1}\right) \\
G\left(\widetilde{\psi}_{2}\right) \\
G\left(\widetilde{\psi}_{3}\right) \\
G\left(\widetilde{\psi}_{4}\right)
\end{array}\right] .
$$

Values of $R_{i}$ can be determined by Eq. (27) or by applying Cramer's rule, and unknown parameters of the second spherical four-bar linkage can be found as shown in Eq. (28).

$$
\bar{R}=\left[g_{i, j}\right]^{-1} \cdot \bar{G}
$$

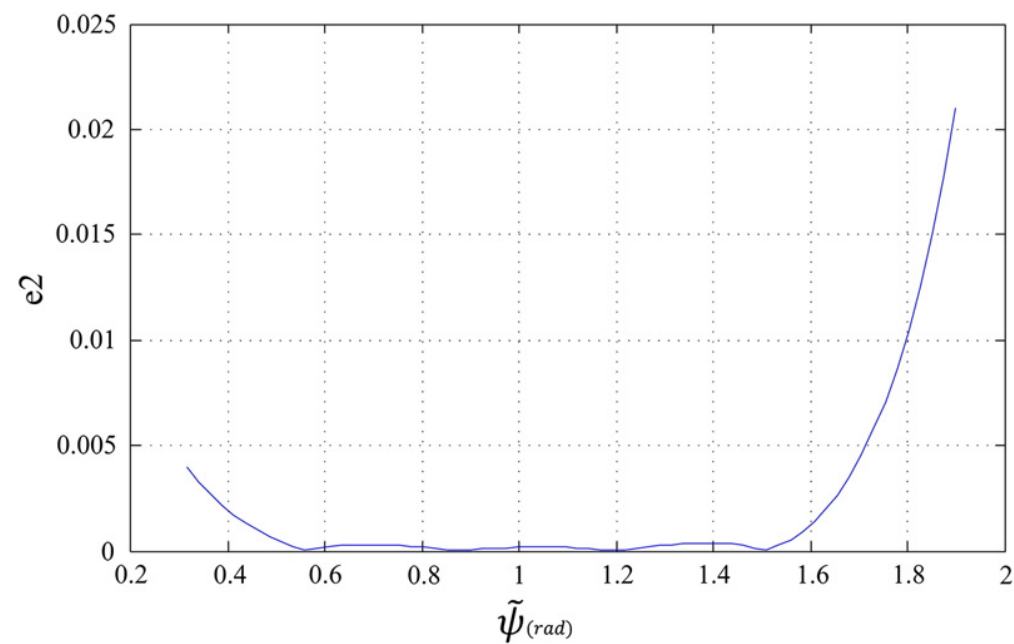

Fig. 8. Calculated error for the second spherical linkage of example \#1. 


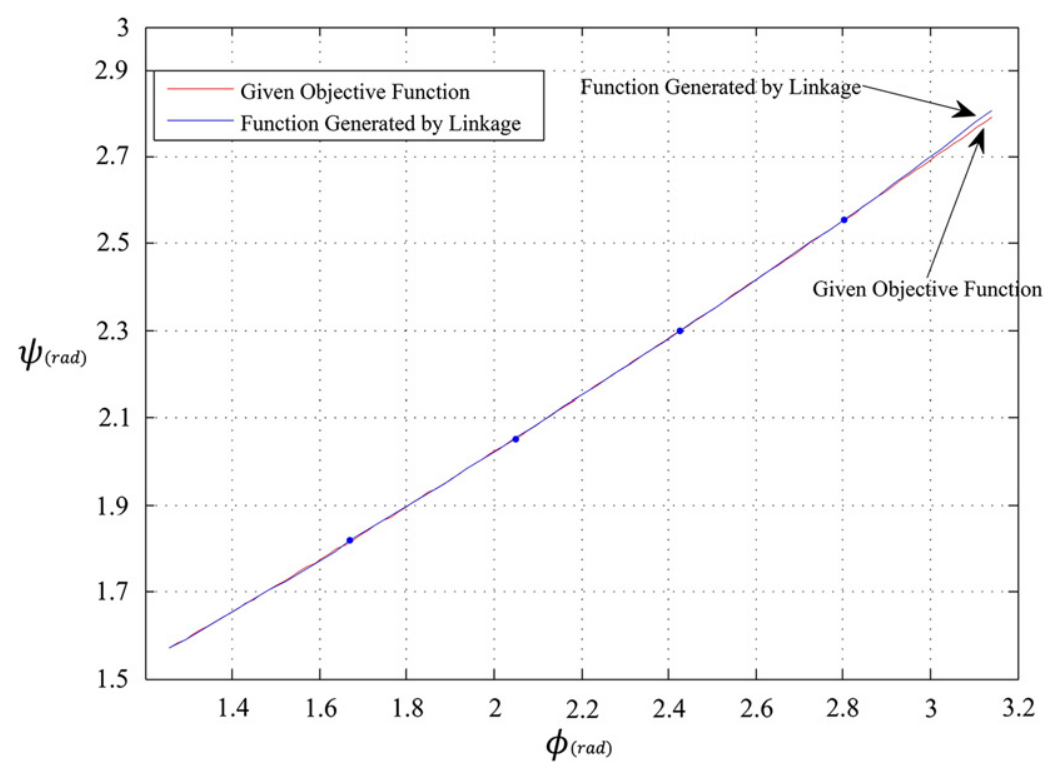

Fig. 9. Function generated by linkage and given objective function $y=x^{1.3}$ of example \#1.

$$
\begin{aligned}
& \alpha_{8,14}=\cos ^{-1}\left(-R_{2}\right), \quad \alpha_{14,12}=\cot ^{-1}\left(-R_{1} / \sin \left(\alpha_{8,14}\right)\right), \quad \alpha_{8,10}=-\cot ^{-1}\left(-R_{3} / \sin \left(\alpha_{8,14}\right)\right) \\
& \alpha_{12,10}=\cos ^{-1}\left(\sin \left(\alpha_{8,10}\right) \cdot \sin \left(\alpha_{14,12}\right) \cdot\left(\cos \left(\alpha_{8,14}\right) \cdot \cot \left(\alpha_{8,10}\right) \cdot \cot \left(\alpha_{14,12}\right)+R_{0}\right)\right)
\end{aligned}
$$

Generated function can be calculated by using the construction parameters determined in Eq. (28) and the input $\widetilde{\psi}$ as in Eq. (15), where $\bar{d}=\left\{\alpha_{8,14}, \alpha_{14,12}, \alpha_{8,10}, \alpha_{12,10}\right\}$.

\section{Numerical examples}

Equally spaced precision points are used to find the mechanism that will generate minimum error. Then, precision points are shifted within a range of $\pm 0.5 \delta$ with $0.1 \delta$ steps, which resulted in 10 different sets of precision points. After each selection of precision points, $P_{i} \& R_{i}$ values are controlled if they are real numbers, and values of $P_{2} \& R_{2}$ are checked if they are within the range \pm 1 . If all these values meet the criteria stated in the previous sentence, then the results of the synthesis procedure are added to the list that contains the possible spherical four-bar linkages with their construction parameters and related errors.

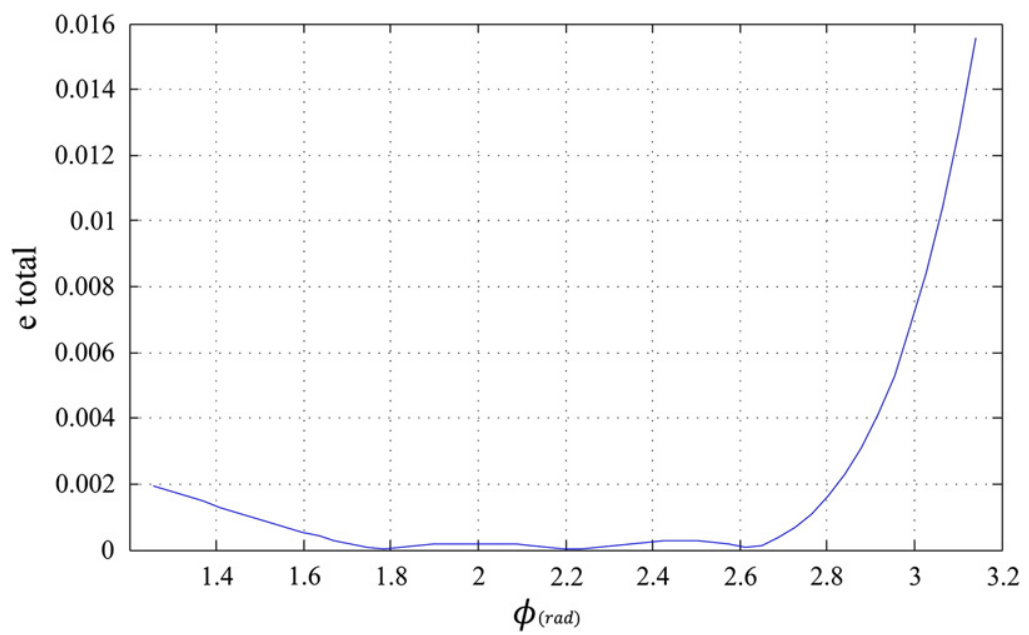

Fig. 10. Total error of the double-spherical six-bar linkage of example \#1. 
Table 3

Precision points and designed construction parameters for the first spherical four-bar linkage of example \#2.

\begin{tabular}{lllllll}
\hline$i$ & $x_{i}$ & $\dot{y}_{i}$ & $\phi_{i}$ rad & $\bar{\psi}_{i}(\mathrm{rad})$ & $P_{i}$ & First four-bar parameters (rad) \\
\hline 1 & 1.2800 & 4.6460 & 1.7844 & 0.5845 & 0.6011 & $\alpha_{2,8}=0.3755$ \\
2 & 1.4800 & 5.9062 & 2.1614 & 0.8415 & 0.3772 & $\alpha_{2,4}=-1.1245$ \\
3 & 1.6800 & 7.5082 & 2.5384 & 1.1682 & -0.9303 & $\alpha_{8,6}=-0.7714$ \\
4 & 1.8800 & 9.5448 & 2.9154 & 1.5835 & -0.1755 & $\alpha_{4,6}=0.8421$ \\
\hline
\end{tabular}

Finally, best approximation is selected with respect to the criteria to have minimum error and to be constructible. The error can be calculated by the difference between the selected or desired objective function and the generated function (the generated functions are simplified using MATLAB® by substituting the calculated construction parameters in function generation) for the first linkage and the second linkage as shown in Eqs. (30) and (31) respectively.

The error for the first four-bar linkage is calculated as

$$
e_{1}=\left|\left(b_{1}^{\prime} h\left(\frac{\phi-\hat{a}_{2}}{a_{1}}\right)+b_{2}^{\prime}\right)-h(\phi, \bar{c})\right| .
$$

The error for the second four-bar linkage is calculated as

$$
e_{2}=\left|\left(b_{1} g\left(\frac{\tilde{\psi}-b_{2}}{b^{\prime}{ }_{1}}\right)+b_{2}\right)-g(\widetilde{\psi}, \bar{d})\right| \text {. }
$$

\subsection{Example \#1}

Let's consider that the given objective function for the synthesis of the whole mechanism is $y=x^{1.3}$ and $90^{\circ} \leq \psi \leq 160^{\circ}$ is the desired range for the output of the double-spherical six-bar linkage. The desired input range of the mechanism is $72^{\circ} \leq \phi \leq 180^{\circ}$.

For the first four-bar linkage the arbitrary function $\hat{y}=x^{0.8}$ is selected as the objective function and $18^{\circ} \leq \widetilde{\psi} \leq 108^{\circ}$ is selected as the arbitrary range for the output of first spherical four-bar linkage. $72^{\circ} \leq \phi \leq 180^{\circ}$ is the desired range for the mechanism input as it was previously set. The minimum error for the first four-bar linkage is calculated to be $e_{1}=0.0537$. The corresponding precision points and the parameters are shown in Table 1.

In Fig. 5, selected objective function and function generated by the linkage are shown for the first spherical four-bar linkage. Precision points are indicated with blue dots in Fig. 5. The calculated error is shown in Fig. 6.

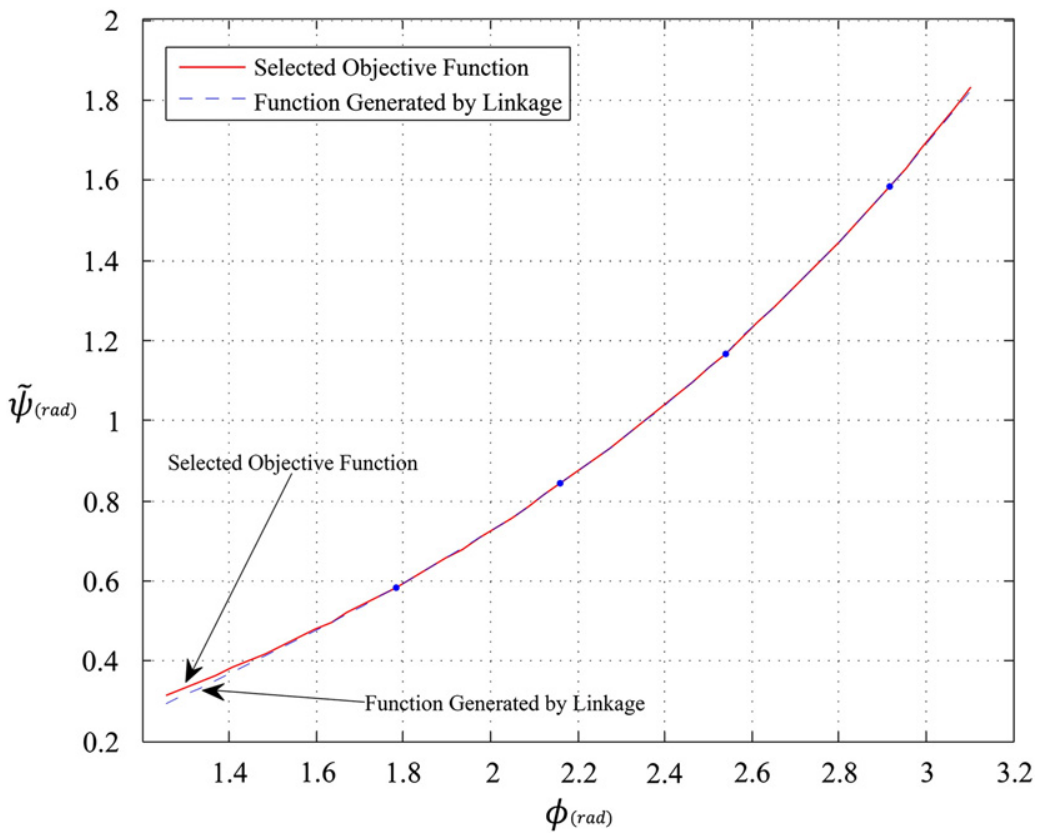

Fig. 11. Selected objective function, $y=e^{1.2 x}$ and function generated by linkage of example \#2. 


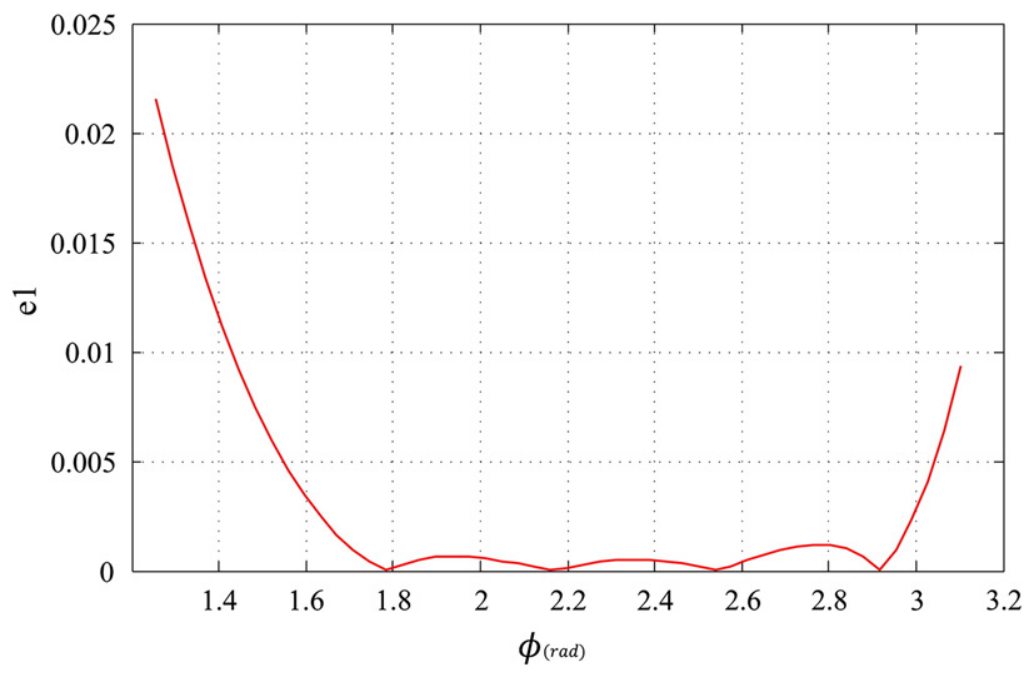

Fig. 12. Calculated error for the first spherical linkage of example \#2.

The objective function of the second spherical mechanism is dependent on the overall mechanism objective function and the arbitrary objective function of the first spherical mechanism. Thus, the second spherical mechanism's function is calculated to be $y=y^{1.625}$. Therefore, the resultant objective function becomes equal to the set function for the whole mechanism, $y=\left(x^{0.8}\right)$ ${ }^{1.625}=x^{1.3}$. The desired range of the input for the second spherical mechanism is selected to be equal to the range of output for the first spherical mechanism as $18^{\circ} \leq \widetilde{\psi} \leq 108^{\circ}$. The range of output for the second spherical mechanism is already specified at the beginning as the range of output for the whole mechanism, $90^{\circ} \leq \psi \leq 160^{\circ}$. The minimum error for the second four-bar spherical linkage is calculated to be $e_{2}=0.0993$. The corresponding precision points and the parameters are shown in Table 2 .

In Fig. 7, the given function and objective function are shown for the second spherical four-bar linkage. Precision points are indicated with blue dots in Fig. 7. The error graph is depicted in Fig. 8.

The overall comparison between the generated function and given objective function is presented in Fig. 9 between the range of input and the range of output. Precision points are indicated with blue dots in Fig. 9. The minimum error for the double-spherical six-bar linkage is calculated to be 0.0738 . The error graph with respect to the input range for the whole mechanism can be observed in Fig. 10.

\subsection{Example \#2}

For the second example, let's consider that the given objective function for the synthesis of the whole mechanism is $y=e^{2 x}$ and $90^{\circ} \leq \psi \leq 160^{\circ}$ is the desired range for the output of the double-spherical six-bar linkage. The desired input range of the mechanism is $72^{\circ} \leq \phi \leq 180^{\circ}$.

For the first four-bar linkage the arbitrary function $\hat{y}=e^{1.2 x}$ is selected as the objective function and $18^{\circ} \leq \widetilde{\psi} \leq 108^{\circ}$ is selected as the arbitrary range for the output of the first spherical four-bar linkage. $72^{\circ} \leq \phi \leq 180^{\circ}$ is the desired range for the mechanism input as it was previously set. The minimum error for the first four-bar linkage is calculated to be $e_{1}=0.1546$. The corresponding precision points and the parameters are shown in Table 3.

In Fig. 11, selected objective function and the function generated by the linkage are shown for the first spherical four-bar linkage of example \#2. Precision points are indicated with blue dots in Fig. 11. The calculated error is shown in Fig. 12.

As mentioned for the first example, the objective function of the second spherical mechanism is dependent on the overall mechanism objective function and the arbitrary objective function of the first spherical mechanism. Thus, the second spherical mechanism's objective function is calculated to be $y=y^{(2 / 1.2)}$. Therefore, the resultant function becomes equal to the set function for the whole mechanism, $y=\left(e^{1.2 x}\right)^{(2 / 1.2)}$. The desired range of the input for the second spherical mechanism is selected to be equal to the range of output for the first spherical mechanism as $18^{\circ} \leq \widetilde{\psi} \leq 108^{\circ}$. The range of output for the second spherical is

Table 4

Precision points and designed construction parameters for the second spherical four-bar linkage of example \#2.

\begin{tabular}{|c|c|c|c|c|c|c|}
\hline$i$ & $\dot{y}_{i}$ & $y_{i}$ & $\bar{\psi}_{i}(\mathrm{rad})$ & $\psi_{i}(\mathrm{rad})$ & $R_{i}$ & Second four-bar parameters ( $\mathrm{rad}$ ) \\
\hline 1 & 4.5526 & 12.5054 & 0.5655 & 1.7032 & -3.3772 & $\alpha_{8,14}=1.5969$ \\
\hline 2 & 6.0932 & 20.3271 & 0.8796 & 1.9056 & 3.8417 & $\alpha_{14,12}=-0.2546$ \\
\hline 3 & 7.6338 & 29.5961 & 1.1938 & 2.1455 & 0.0261 & $\alpha_{8,10}=0.1952$ \\
\hline 4 & 9.1744 & 40.2065 & 1.5080 & 2.4201 & -5.0564 & $\alpha_{12,10}=1.4302$ \\
\hline
\end{tabular}




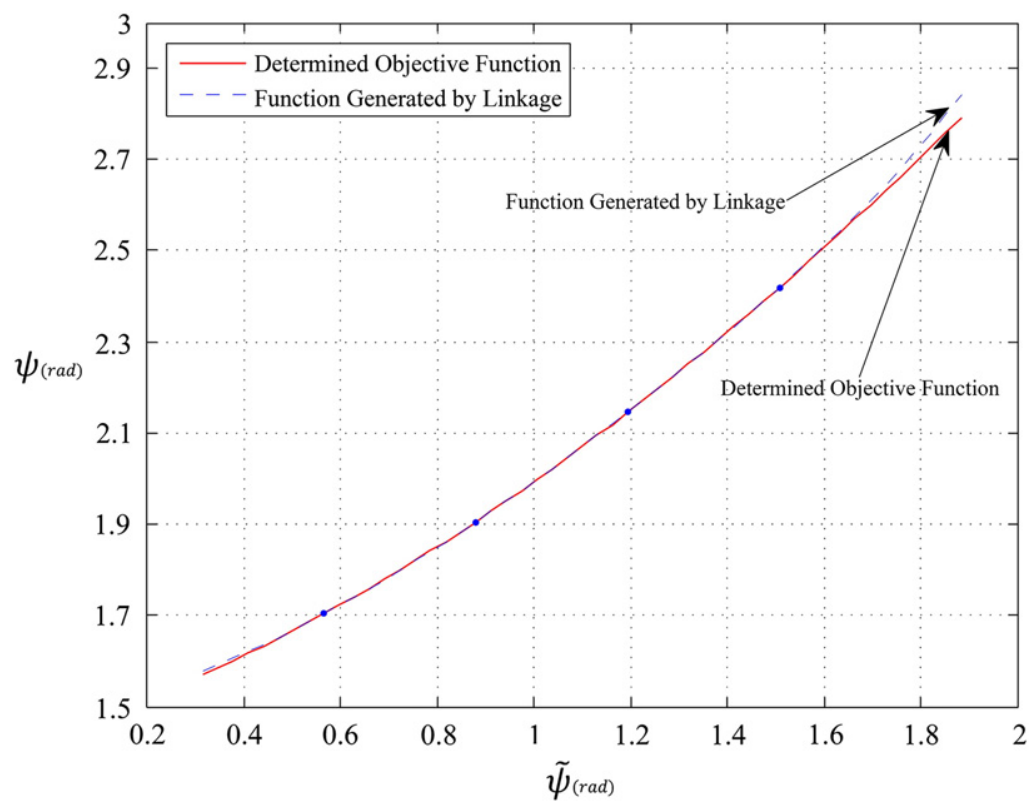

Fig. 13. Selected objective function, $y=\dot{y}^{(2 / 1.2)}$ and function generated by the second linkage of example \#2.

already specified at the beginning as the range of output of the whole mechanism, $90^{\circ} \leq \psi \leq 160^{\circ}$. The minimum error for the second four-bar spherical linkage is calculated to be $e_{2}=0.2342$. The corresponding precision points and the parameters are shown in Table 4.

In Fig. 13, the determined objective function and the function generated by linkage are shown for the second spherical four-bar linkage of example \#2. Precision points are indicated with blue dots in Fig. 13. The error graph is given in Fig. 14.

For the second example, the overall comparison between the objective function and function generated by the whole mechanism is presented in Fig. 15 between the range of input and the range of output. In Fig 15, the precision points are indicated with blue dots. The minimum error for the double-spherical six-bar linkage is calculated to be 0.0744 . The error graph with respect to the input range for the whole mechanism can be observed in Fig. 16.

\section{Conclusions}

The spherical four-bar and the planar four-bar linkages contain only revolute joints and they have been well studied in mechanism design. However, no study has been carried out on the synthesis construction design of serially connected spherical

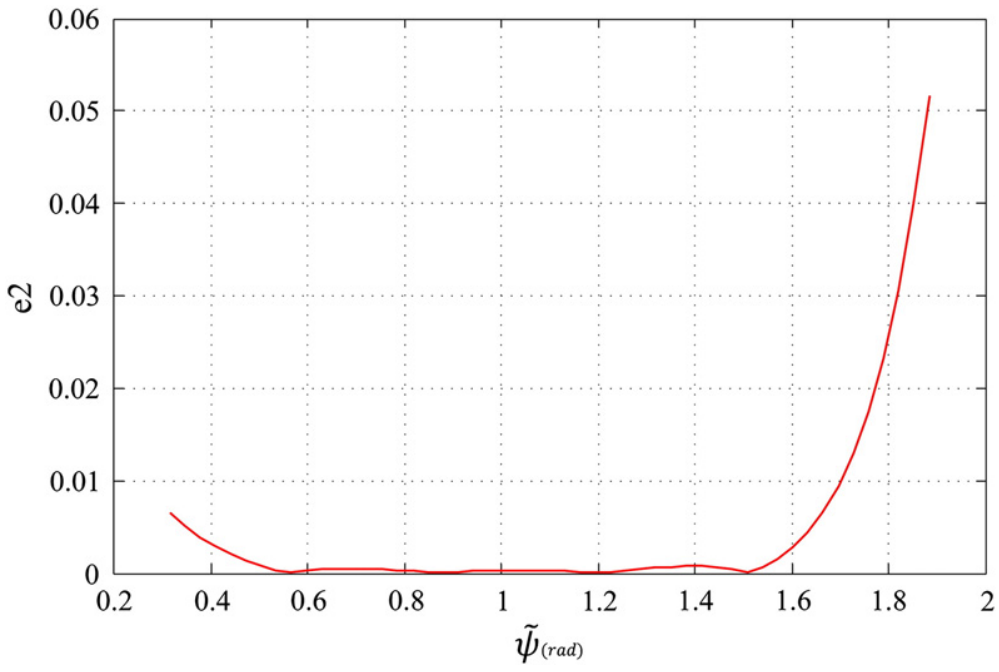

Fig. 14. Calculated error for the second spherical linkage of example \#2. 


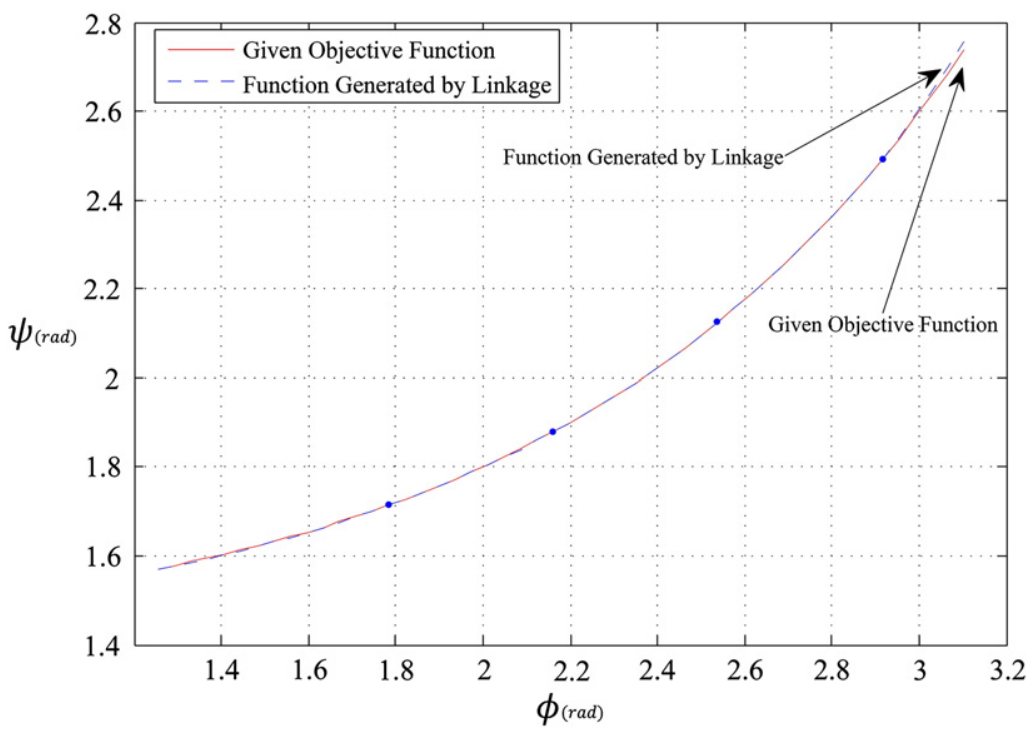

Fig. 15. Function generated by linkage and given objective function $y=e^{2 x}$ of example \#2.

four-bar mechanisms. The comparative structural syntheses of planar and spherical six-bar mechanisms are discussed. Kinematic synthesis of the double-spherical six-bar mechanism is formulated by separating the mechanism into two spherical four-bar linkages by the addition of a passive revolute joint. Decomposition method is defined to share the overall mechanism's function between two spherical four-bar linkages which work as a function of function system. Transformation of unit vector equation is used to find the objective functions of the two spherical four-bar linkages. The limit positions and objective function for the relation of the input and output link for the double-spherical six-bar mechanism are given. The limit positions for the output link and the objective function that defines the relation between the input and output link for the first spherical four-bar linkage are selected arbitrarily to design the construction parameters of the first spherical linkage. The calculated construction parameters are checked with respect to constructability and for minimum error. First spherical four-bar linkage is designed with respect to the calculated construction parameters and then, the output angle of the first spherical four-bar linkage is used as the input of the second spherical four-bar linkage. This is realized through the virtual (passive) joint inserted between them. Finally, the synthesis procedure is applied for the second spherical four-bar mechanism and the last four construction parameters are designed for the smallest error and constructability. The overall error of the mechanism's function generation as a result of the decomposition method is calculated and presented to be within the same ranges of errors as two spherical four-bar linkage errors.

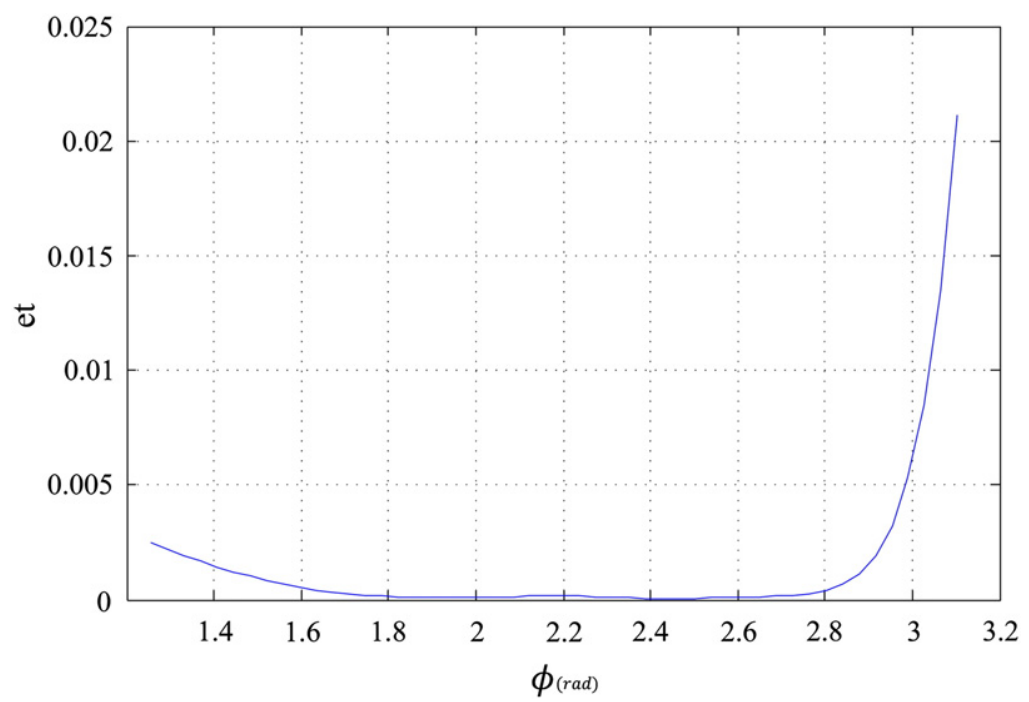

Fig. 16. Total error of the double-spherical six-bar linkage of example \#2. 
In order to validate that the procedure holds for different functions that have more nonlinear characteristics, two example studies with different nonlinearity characteristics are carried out. It is shown that for both numerical examples, the kinematic synthesis method with the decomposition method could be applied and generated functions were able to match the desired objective function with bounded errors at similar error ranges.

It should be noted that during the design of the first four-bar mechanisms, the inputs are within the range specified for the input of the whole mechanism and the outputs for the second four-bar mechanisms are selected to have the same range as the whole mechanism's output.

The synthesis procedure carried out in this study is for four precision points. This results in four linear equations to solve for each spherical loop. This method can be extended for five and even six precision points since relative studies on spherical four-bar mechanisms are available $[11,12]$. Kinematic synthesis of double-spherical six-bar mechanism with five or six precision points is regarded as a future study, in which one can foresee that in addition to linear equations, there will be a total of four nonlinear equations in the case of five precision points and 12 nonlinear equations in the case of six precision points to solve for.

\section{Appendix A. Supplementary data}

Supplementary data to this article can be found online at http://dx.doi.org/10.1016/j.mechmachtheory.2013.10.013.

\section{References}

[1] D.A. Ruth, J.M. McCarthy, The design of spherical 4R linkages for four specified orientations, Mech. Mach. Theory 34 (5) (1999) 677-692.

[2] J.M. McCarthy, Geometric Design of Linkages, first ed. Springer, New York, 2000.

[3] J.J. Cervantes-Sanchez, J.I.H. Medellin-Castillo, A robust classification scheme for spherical 4R linkages, Mech. Mach. Theory 37 (10) (2002) $1145-1163$.

[4] I.H. Medellin-Castillo, J.J. Cervantes-Sanchez, An improved mobility analysis for spherical 4R linkages, Mech. Mach. Theory 40 (8) (2005) $931-947$.

[5] Tong Shih-Hsi, C.H. Chiang, Syntheses of planar and spherical four-bar path generators by the pole method, Mech. Mach. Theory 27 (2) (1992) 143-155.

[6] K. Farhang, Y.S. Zarqar, Design of spherical 4R mechanisms: function generation for the entire motion cycle, J. Mech. Des. Trans. ASME 121 (4) (1999) 521-528.

[7] J. Denavit, R.S. Hartenberg, Kinematic Synthesis of Linkages, McGraw-Hill, New York, 1964

[8] J.R. Zimmerman, Four-precision-point synthesis of the spherical four-bar function generator, Mech. Mach. Theory 2 (2) (1967) 133-139.

[9] A.P. Murray, J.M. McCarthy, A linkage map for spherical four position synthesis, ASME Tech. Conf., Boston, MA, 1995, pp. 833-844.

[10] R.I. Alizade, Synthesis of four-bar spherical mechanism on five parameters, J. Mech. Eng, 6, Russian Academy of Science (ANR), 1994. (in Russian).

[11] R.I. Alizade, O. Kilit, Analytic synthesis of function generating spherical four-bar mechanism for five precision points, Mech. Mach. Theory 40 (7) (2005) 863-878.

[12] R.I. Alizade, E. Gezgin, Synthesis of function generating spherical four bar mechanism for the six independent parameters, Mech. Mach. Theory 46 (9) (2011) 1316-1326.

[13] N. Makhsudyan, R. Djavakhyan, V. Arakelian, Comparative analysis and synthesis of six-bar mechanisms formed by two serially connected spherical and planar four-bar linkages, Mech. Res. Commun. 36 (2) (2009) 162-168.

[14] Q.S. Shen, K. Russell, R.S. Sodhi, Expended 4R spherical motion generation with a prescribed rigid-body load, Proc. 10th Int. Conf. Theory Mach. Mech., Liberec, Czech Republic, 2008, pp. 543-548.

[15] K. Brunnthaler, H.P. Schröcker, M. Husty, Synthesis of spherical four-bar mechanisms using spherical kinematic mapping, in: J. Lenancic, B. Roth (Eds.), Advances in Robot Kinematics: Mechanism and Motion, Springer, Netherlands, 2006, pp. 377-384.

[16] B. Hong, A.G. Erdman, A method for adjustable planar and spherical four-bar linkage synthesis, J. Mech. Des. 127 (3) (2005) $456-463$.

[17] D. Chablat, J. Angeles, The computation of all 4R serial spherical wrists with an isotropic architecture, J. Mech. Des. 125 (2) (2003) $275-280$.

[18] R.I. Alizade, C. Bayram, E. Gezgin, Structural synthesis of serial platform manipulators, Mech. Mach. Theory 42 (5) (2007) 580-599.

[19] J.E. Baker, Displacement-closure equations of the unspecialized double-Hooke's-joint linkage, Mech. Mach. Theory 37 (2002) 1127-1144.

[20] L. Cui, J.D. Dai, The axis constraint equation and a general 6R double-spherical overconstrained mechanism, in: A. Kecskemethy, A. Muller (Eds.), Computational Kinematics, Springer, 2009, pp. 233-240.

[21] R. Alizade, Structural synthesis of robot manipulators, Proc. Int. Symp. Mech. Mach. Sci., Izmir, Turkey, 2010, pp. 11-32.

[22] F. Freudenstein, R. Alizade, On the Degree of Freedom of Mechanisms with Variable General Constraint, IV. World IFTOMM Congress, England, $1975.51-56$. 